\title{
Molecular docking, molecular dynamic and drug-likeness studies of natural flavonoids as inhibitors for SARS-CoV-2 main protease (Mpro)
}

\author{
Listiana OKTAVIA 1,2* (D), Praptiwi PRAPTIWI 1,2 (D), Andria AGUSTA 1,2 (D) \\ 1 Research Center for chemistry, Indonesian Institute of Sciences, PUSPITEK Serpong, Tangerang Selatan, Banten \\ 15314, Indonesia. \\ 2 Research Center for Chemistry, National Research and Innovation Agency (BRIN), PUSPITEK Serpong, Tangerang \\ Selatan, Banten 15314, Indonesia. \\ * Corresponding Author. E-mail: listiana.oktavia@brin.go.id / oktavialisti@gmail.com (LO) ; Tel. +62 2187907604
}

Received: 19 May 2021 / Revised: 05 November 2021 / Accepted: 14 November 2021

\begin{abstract}
The emergence of the global pandemic COVID-19 lead to a huge demand for the therapeutic agent to combat the disease. Since the FDA approval of some of HIV-1 main protease inhibitors such as ritonavir lopinavir to treat COVID-19, the investigation of anti-HIV inhibitor to inhibit SARS-CoV-2 main protease (Mpro) is getting considerably much attention. This study evaluates the potency of sixteen selected natural flavonoids which were previously reported active to block HIV-1 protease as potential inhibitors of SARS-CoV-2 Mpro. The molecular docking and dynamic study were completed to know the binding affinity and stability of the protein-ligand complex via docking study along with molecular dynamic simulations. Moreover, drug-likeness was also evaluated through via ADMET evaluation. This study revealed robinin (6), a flavonol molecule with linked to galactose-rhamnose at C 3 and rhamnose molecule at C7, exhibited the highest binding affinity $(-9 \mathrm{kcal} / \mathrm{mol})$ among others. The amino acids that interacted with robinin were Asn142; Gly143; Arg188; Thr190. The binding affinity of robinin surpassed the binding affinity of ritonavir $(-7.7 \mathrm{kcal} / \mathrm{mol})$ and lopinavir $(-8.2 \mathrm{kcal} / \mathrm{mol})$. The replacement of the hydroxyl group from the flavonoid skeleton at C$7, \mathrm{C}-4^{\prime}$ was proposed to affect the binding affinity. The free hydroxyl group particularly in A ring and the position of the hydroxyl group were important to improve the binding affinity. The molecular dynamic simulation showed the stability of Mpro-robinin during the simulation period. The ADME evaluation referring to Lipinski`s rule of 5 revealed that the flavonoids $(\mathbf{2}, \mathbf{5}, \mathbf{6 , 9}, \mathbf{1 0}, \mathbf{1 3}, \mathbf{1 4}, \mathbf{1 5})$ show low oral bioavailability and absorption. Robinin exhibited a good druglikeness score (value:1) with an unconcerned level of acute toxicity. From this study, it was concluded that robinin showed the most potent natural flavonoids studied to inhibit SASR-CoV-2 Mpro by both docking study and ADME/tox properties evaluation.
\end{abstract}

KEYWORDS: Anti-viral; COVID-19; Docking; Flavonoids; Mpro.

\section{INTRODUCTION}

Coronavirus disease -2019 (COVID-19) caused by a single causative agent SARS-CoV-2 is a single was firstly emerged in Wuhan China, at the end of 2019 and since then has been spreading rapidly all around the globe led to the global pandemic [1]. As written in the WHO situation report, by the end of April 2021, the total case of Covid-19 globally has reached $145 \mathrm{M}$ causing 3.11 M death tolls. The rapid human transmitter of SARS-CoV-2 made a great escalation of the current pandemic. According to the previous study, this virus is classified into B-coronavirus with positive-sense RNA [2]. This virus is transmitted via inhalation of infected droplets or by interaction with the contaminated surfaces of infected people [3]. SARS-CoV-2 consists of many nonstructural proteins, some of the major concerned proteins including coronavirus main protease (3CLpro or Mpro), RDRP (RNA-dependent RNA polymerase), ACE2, spike protein [4]. The utilization of the main protease of the virus as a drug target had been investigated since the protease exhibits an essential role in viral replication [5]. Due to the highly conserved area, a mutation in Mpro genome is frequently causing a lethal effect to the virus and the catalytic activity of this enzyme could be modified by the fixation of molecular

How to cite this article: Oktavia L, Praptiwi P, Agusta A. Molecular docking, molecular dynamic and drug-likeness studies of natural flavonoids as inhibitors for SARS-CoV-2 main protease (M $\left.{ }^{\text {pro }}\right)$. J Res Pharm. 2021; 25(6): 998-1009. 
binding in the pocket. [6], [7]. Moreover, it has been reported that the unique structure and catalytic characteristics of SARS-CoV-2 Mpro make it a promising target for anti-viral development [2].

While the world is in the race to reduce the spread of COVID-19 by conducting vaccination, a large effort is still undergoing to find effective cures for treating the COVID-19. Most of the current FDA-approved drugs for treating severe Covid-19 cases are possessing a wide range of antiviral, antiparasitic, and antiinflammatory properties such as marboxil, darunavir, favipiravir, lopinavir, oseltamivir, remdesivir, ritonavir [8], [9]. Some of the mentioned drugs, lopinavir and ritonavir which also are known as HIV main protease inhibitors, are reported to bind in the target site of Mpro Covid-19 [10]. The spatial structure of lopinavir and ritonavir in the binding pocket of SARS-CoV Mpro and 2019-nCOv Mpro were known to be conserved [10]. Amino acid Thr24, Asn28, and Asn119 were pictured to form the binding pocket for lopinavir/ritonavir in the spatial structure of both SASR-Cov Mpro and 2019-nCov Mpro [11]

Recently, HIV-1 protease inhibitors (PIs) are greatly evaluated to be developed for SARS-CoV-2 inhibitors. Following the FDA approval for covid-19 treatments, some PIs including lopinavir and ritonavir were also documented to be active against SARS-CoV and MERS by in vitro experiments [12]. Considering the 94.4\% similarity in amino acid sequences between SARS CoV and SARS CoV-2 [13], investigating the efficacy of PIs against SARS-CoV-2 is considered relevant. Thought some HIV inhibitors such as lopinavir and ritonavir have been approved to be clinically used to treat COVID-19, both drugs possess high cytotoxicity against primary myeloma cells [14]. Ritonavir was reported to cause endothelial mitochondrial DNA damage and cell death through the necrosis pathway [15]. Therefore, there is an urgent need to find alternative drug candidates for Covid-19 treatment with a high inhibitory effect on viral replication with reduced side effect properties.

Natural products have proven to generate many lead compounds for treating disease. Natural products can be a great source of anti-viral compounds. One of the prominent natural products with various biological activities such as anti-HIV, anti-inflammatory, and cardiovascular protective effects [2], [16], [17] is flavonoids. Natural flavonoids classified as flavanol or flavone exhibited simultaneous inhibition toward Reverse Transcriptase (RT) and protease. Meanwhile, the flavanol, isoflavone, or flavanone group were displayed less inhibition to RT or protease [16]. Meanwhile, the flavanol, isoflavone, or flavanone group were displayed less inhibition to RT or protease [16]. Flavonoids are considered important PIs due to their strong antiviral activity and low toxicity [18]. Xu et. al [19] reported a wide variety of flavonoids compounds as an inhibitor of HIV-main protease. Some of them such as quercetin, rutin, robinin, kaempferol, morin, scutellarein, luteolin, etc, were monitored to exhibit the inhibition activity by more than $50 \%$ against HIV-1 protease. One of biflavonoid, amentoflavone, was known to be registered in European Patent EP1245230 due to its strong inhibitor for HIV-1 protease [16]. Flavanols are considered as a promoter to induce the inhibition of many enzymes involving the coronavirus infection [2]. The basic skeleton of flavonol shows the activity as antiviral which is confirmed by Owis et.al [20].

In the present study, sixteen selected natural flavonoids (Figure 1), previously reported active to block HIV protease, were evaluated as potential inhibitors for SARS-CoV-2 inhibitors in silico study. The selected flavonoids representing flavonol (1-6, 9), flavone $\mathbf{( 7 , 1 0 , 1 1 , 1 2 , 1 4 , 1 5 ) , ~ c h a l c o n e ~}(\mathbf{8})$, catechols $(\mathbf{1 3 , 1 6})$ groups were chosen according to their in vitro PIs activity with inhibition activity of more than $60 \%$ as reported by Xu et. al [19] and Zakaryan et. al [21]. The selected compounds would be docked to understand the binding affinity and the interaction between selected flavonoids and the SARs-CoV-2 Mpro. In addition, the stability of the protein-ligand complex during some period in the water environment will also be simulated through molecular dynamic simulations. The effect of particular functional groups in the flavonoid skeleton on the binding affinity with protein will also be analyzed. Furthermore, the drug-likeness of flavonoids would be evaluated to predict the ADME properties of tested flavonoids. This study hopefully could be contributed to embark the study of the development of COVID-19 cure-based natural products. 

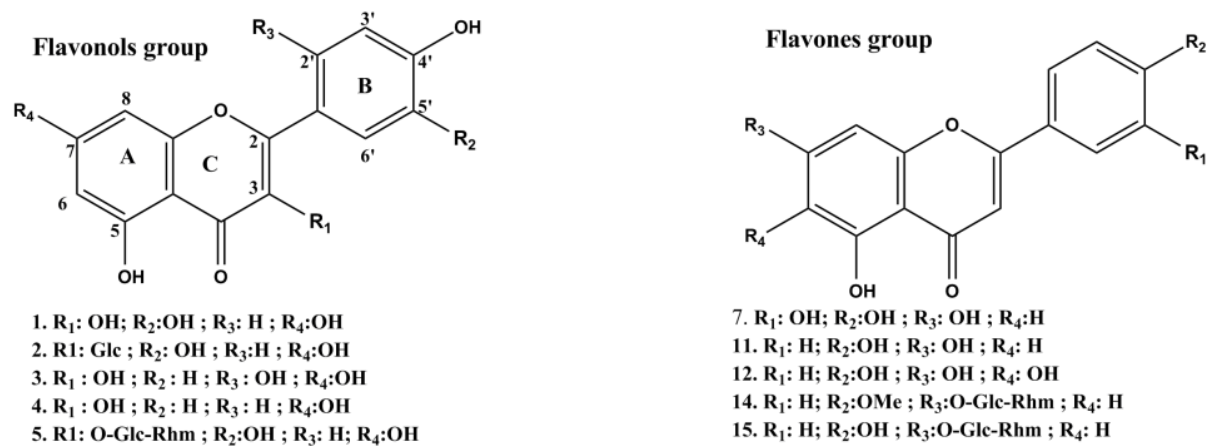

1. $\mathrm{R}_{1}: \mathrm{OH} ; \mathrm{R}_{2}: \mathrm{OH} ; \mathrm{R}_{3}: \mathrm{H} ; \mathrm{R}_{4}: \mathrm{OH}$
2. $\mathrm{R} 1: \mathrm{Glc} ; \mathrm{R}_{2}: \mathrm{OH} ; \mathrm{R}_{3}: \mathrm{H} ; \mathrm{R}_{4}: \mathrm{OH}$

2. R1: Glc; $\mathrm{R}_{2}: \mathrm{OH} ; \mathrm{R}_{3}: \mathbf{H} ; \mathbf{R}_{4}: \mathrm{OH}$
3. $\mathbf{R}_{1}: \mathrm{OH} ; \mathbf{R}_{2}: \mathbf{H} ; \mathbf{R}_{3}: \mathrm{OH} ; \mathbf{R}_{4}: \mathrm{OH}$

4. $\mathrm{R}_{1}: \mathrm{OH} ; \mathrm{R}_{2}: \mathbf{H} ; \mathrm{R}_{3}: \mathbf{H} ; \mathbf{R}_{4}: \mathrm{OH}$

5. R1: O-Gle-Rhm $; \mathrm{R}_{2}: \mathrm{OH} ; \mathrm{R}_{3}: \mathrm{H} ; \mathrm{R}_{4}: \mathrm{OH}$

6. R1: O-Gal-Rhm ; $\mathrm{R}_{2}: \mathrm{OH} ; \mathrm{R}_{3}: \mathrm{H} ; \mathrm{R}_{4}: \mathrm{O}-\mathrm{Rhm}$

9. $\mathrm{R}_{1}: \mathrm{OH} ; \mathrm{R}_{2}: \mathrm{OH} ; \mathrm{R}_{3}: \mathrm{H}_{\mathrm{R}_{4}}: \mathrm{OH} ; \mathrm{C}^{3} 3^{\prime}: \mathrm{OH}$
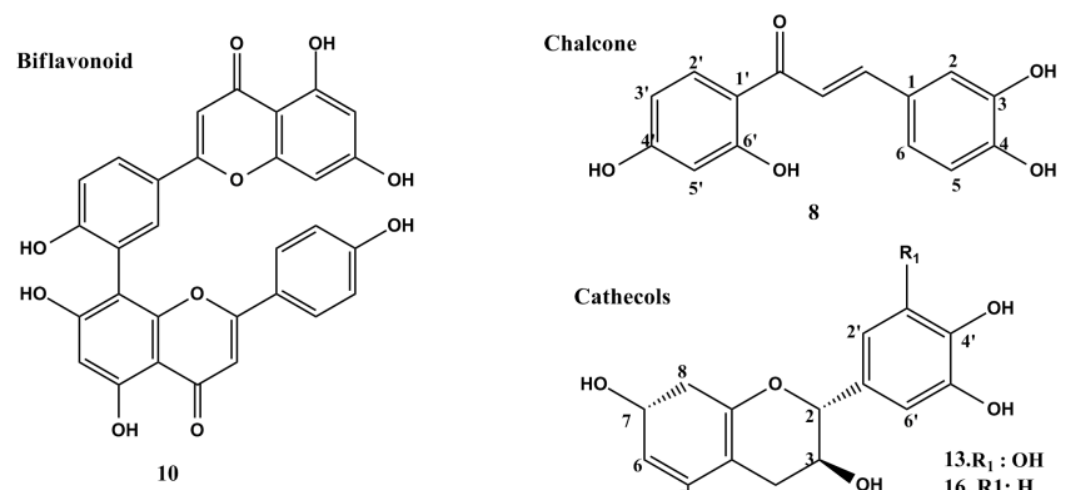

Glc: glucose; Rhm : rhamnose; Gal: galactose

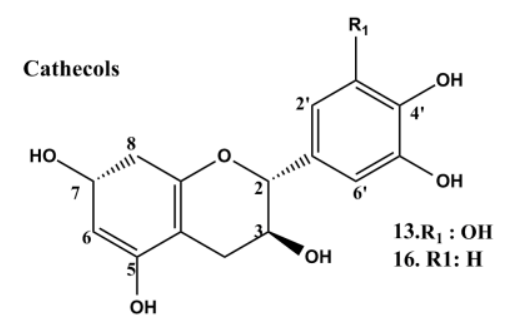

Figure 1. The chemical structure of 16 selected flavonoids for docking study.

\section{RESULTS and DISCUSSION}

\section{1 Molecular Docking Results}

The SASR-CoV-2- Mpro possesses the conserved catalytic dyad formed by His41 and Cys145. The cocrystalized ligand of Mpro (6LU7) identified as N3 (Michael acceptor (peptidyl)) was suitably placed in the substrate-ligand binding pocket of SARS-CoV-2 Mpro [20]. Based on that information, the docking parameters of selected flavonoids were constructed as guided by the N3 coordinate area. The method validation was conducted by re-docking the N3 inhibitor into Mpro using designated parameters (methodology section). The superimposed picture (2.A) of the co-crystallized N3 ligand before and after re-docking showed the good accuracy of the designated experiment parameters. Moreover, the root-mean-square deviation (RMSD) value of N3 ligand before and after redocking was calculated 1.28 $\AA$. The docking parameters are considered to be valid if the RMSD value of re-docked native ligands is below $2 \AA$ [22].

The docking results of selected flavonoids indicated the binding preference like N3 inhibitor. Most dominant hydrogen bonding was formed by ligands and amino acids Leu141, Ser144, His163, and Thr190 as written in table 1 . The binding affinity and amino acid involved in the ligand- Mpro are described in table 1. The affinity binding energy result was ordered as follows: N3>6>2=10>12>15>3=4=11>7>8=9>15=14>5>13. Robinin (6) was shown the highest binding affinity with docking value $-9 \mathrm{kcal} / \mathrm{mol}$. Amino acid involved in the complex formation identified as Asn142; Gly143; Arg188; Thr190 (table 1). The 3D structure of robininMpro complex shows robinin perfectly fits with the protein binding pocket as shown in figure (2.B). Robinin is flavanol that is linked to the neohespridoside (galactose-rhamnose) moiety in ring A at C-3. Robinin (6) and rutin (5) both are substituted by disaccharide C-3 but the docking value for robinin (6) was higher than rutin since the presence of rhamnose at C-7. That findings show that the presence of sugar moiety in the C-7 affects the binding affinity. The bulky structure of gal-rhm might be hinder the hydrogen bonding and electrostatic interaction. That suggestion is supported by Owis et.al [20] that stated the presence of rhamnose unit at C-3 at the rutinoside decrease the binding affinity with $\mathrm{M}^{\mathrm{pro}}$. 
Table 1. Docking result of 16 natural flavonoids and SARS-Cov-2 Mpro (PDB id:6LU7).

\begin{tabular}{|c|c|c|c|c|c|}
\hline No & Ligands & Group & $\begin{array}{l}\text { CID } \\
\text { number }\end{array}$ & $\begin{array}{l}\text { Docking } \\
\text { value } \\
\text { (kcal/mol) }\end{array}$ & Amino acid receptor (hydrogen bonding) \\
\hline 1 & Quercetin & flavonol & 5280343 & -7.6 & gln189; Leu141; Ser144; His163 \\
\hline 2 & Isoquercetin & flavonol-glc & 5280804 & -8.3 & Thr190; His163; leu141; Ser144 \\
\hline 3 & Morin & flavonol & 5281670 & -7.8 & Leu141; Ser144; Asp187;Gln189 \\
\hline 4 & Kaempferol & flavonol & 5280863 & -7.8 & Leu141; Ser144;Gln189 \\
\hline 5 & Rutin & Flavonol-glc & 5280805 & -7.2 & Asn142; glu166; Pro168; Gln189 \\
\hline 6 & Robinin & flavonol-glc & 5281693 & -9 & Asn142; Gly143; Arg188; Thr190 \\
\hline 7 & Luteolin & flavone & 5280445 & 7.5 & Asn142; Arg188; Thr190; Gln192 \\
\hline 8 & Butein & chalcone & 5281222 & -7.4 & $\begin{array}{l}\text { leu141;Gly143;Ser144;Cys145;His163; } \\
\text { Arg188; Thr190;Gln192 }\end{array}$ \\
\hline 9 & Myricetin & flavonol & 5281672 & -7.4 & Leu141; gly143; Ser144; cys145; His163 \\
\hline 10 & Amentoflavone & flavone & 5281600 & -8.3 & Gly143; His164 \\
\hline 11 & Apigenin & flavone & 5280443 & -7.8 & Tyr54; Leu141; Ser144; His163; Asp187 \\
\hline 12 & Scutellarein & flavone & 5281697 & -8.1 & Phe140; Leu141; Ser144; His163; Asp187 \\
\hline 13 & (-)-epigalochatechin & catechols & 72277 & -7 & Thr26; Gln189 \\
\hline 14 & Fortunellin & flavone-glc & 5317385 & -7.2 & Asn142; His163; Glu166 \\
\hline 15 & Rhoifolin & flavone-glc & 5282150 & -7.9 & Ser46; Arg188; Thr190; Gln192 \\
\hline 16 & $(+)$-Catechin & catechols & 9064 & -7.3 & Glu166; Asp187 \\
\hline 17 & \multicolumn{2}{|c|}{ N3 inhibitor (native ligand) } & 182232 & -10 & Gly143; His163;Glu166; Gln189; Thr190 \\
\hline
\end{tabular}
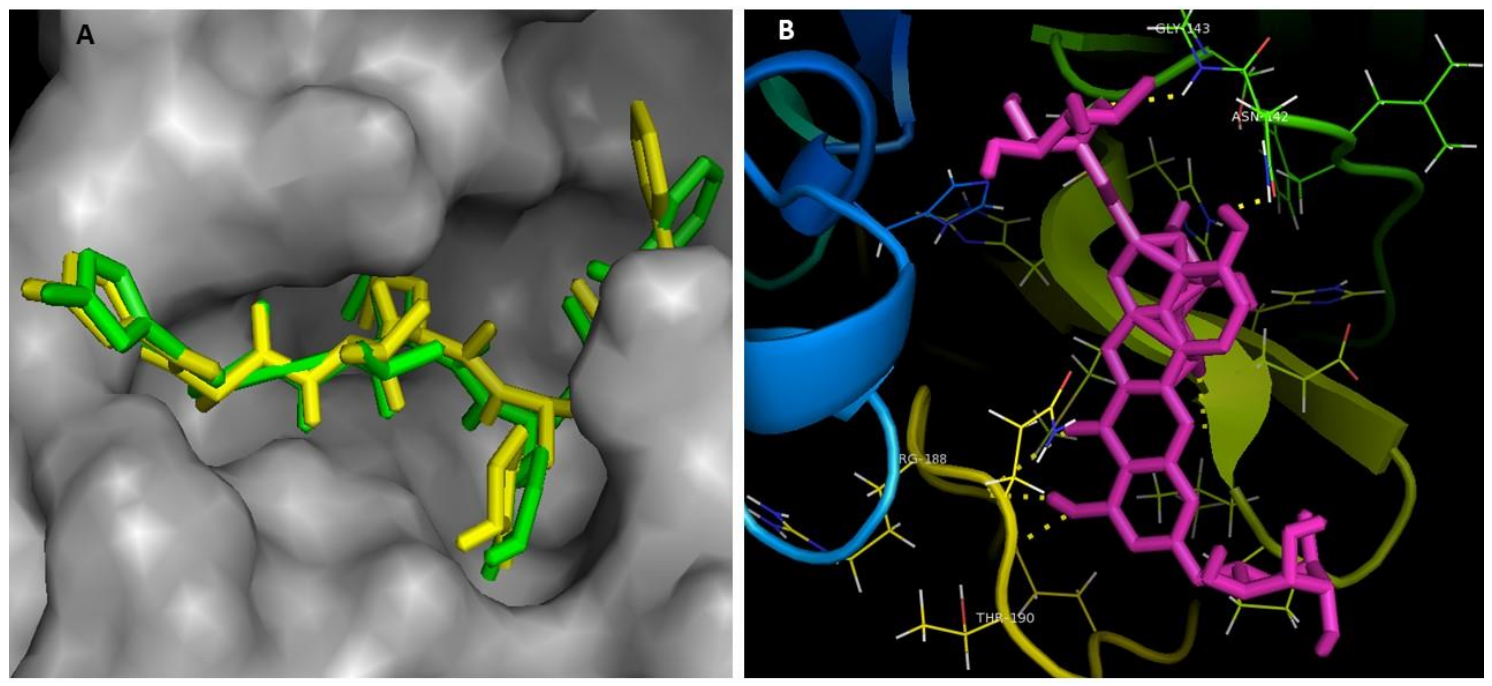

Figure 2. (A). The superimposed picture of native ligand before (green) and after (yellow) re-docking process in the binding pocket of SARS-Cov-2 Mpro. (B). 3D figure of Robinin (magenta) with Mpro, some amino acid interacted with ligand is shown (Asn142, Gly143, Arg, Thr190).

The presence of rhamnose at $\mathrm{C} 7$ changed the molecular conformation of robinin allowed the formation of hydrogen bonding in A ring between the free hydroxyl group at C5 with Arg188 and Thr198 as suggested by Figure (3.A). The substitution of $-\mathrm{OH}$ by glucose at ring A particularly at $\mathrm{C} 3$ of flavonol improved the binding affinity of flavonoids as represented by the docking value of iso-quercetin (2) $(-8.3 \mathrm{kcal} / \mathrm{mol})$ which is higher than quercetin (1) $(-7.6 \mathrm{kcal} / \mathrm{mol})$. While the presence of gal-rhm moiety solely in C-3 was monitored to reduce the binding affinity, as shown by the docking value of rutin (5) $-7.2 \mathrm{kcal} / \mathrm{mol}$ which is lower than quercetin. The addition of $-\mathrm{OH}$ in the $\mathrm{C}^{\prime} 2^{\prime}(\operatorname{morin}(3))$ slightly improved the binding affinity of flavanol compared to the substitution at C-5' (quercetin (1)). The absence of substituents at C-3 of the flavone group resulted in a slight decrement to the binding affinity compared to the flavonol group which is described by 
docking value of luteolin (7) and quercetin (1). In the flavone group, the presence of -OH group at C-5' supplied more binding affinity as displayed by apigenin (11) with docking value $-7.8 \mathrm{kcal} / \mathrm{mol}$. Biflavonoid, amentoflavone (10) consisted of two apigenin (11) molecules exhibited higher binding affinity than its monomer with docking value $-8.3 \mathrm{kcal} / \mathrm{mol}$. The hydrophobic interaction was suggested to promote stabilization to amentoflavone-Mpro complex (Figure 3.B). The addition of $-\mathrm{OH}$ group at A ring (C-6) in apigenin (11, flavone group ) moves the docking value from $-7.8 \mathrm{kcal} / \mathrm{mol}$ (apigenin) into $-8.1 \mathrm{kcal} / \mathrm{mol}$ (scutellarein) (Figure 3.C). The -OH adjunct in the C-5' created the additional hydrogen bonding with Phe-140 which previously did not appear in the apigenin complex. The increase in the binding affinity was shown when -OMe group at C-4' (fortunellin (14)) is changed to -OH (rhoifolin (15)). Both fortunellin and rhoifolin connect to neohesperidoside (glu-rhm) moiety at C-7. The substitution of -OH into rhamnose moiety at C-7 alleviates docking value slightly from -7.8 to $7.9 \mathrm{kcal} / \mathrm{mol}$. The polar substituent at C-7 is important to improve the antiviral activity, as previously described that glycosylation of C-7 of flavonol increase the inhibitory activity against HIV-1 protease [23]. The binding affinity of catechols and chalcone exhibited lower than flavone and flavanols groups. The free hydroxyl group at the C-7 and C-3' are responsible for the hydrogen binding formation. The cleavage of $\mathrm{C}$ ring in chalcone group (butein (8)) might be the reason for the relatively low binding affinity of this compound. This study suggested that the presence of free hydroxyl particularly in the ring B of flavonol and flavone is pivotal to improving the binding affinity with the Mpro.

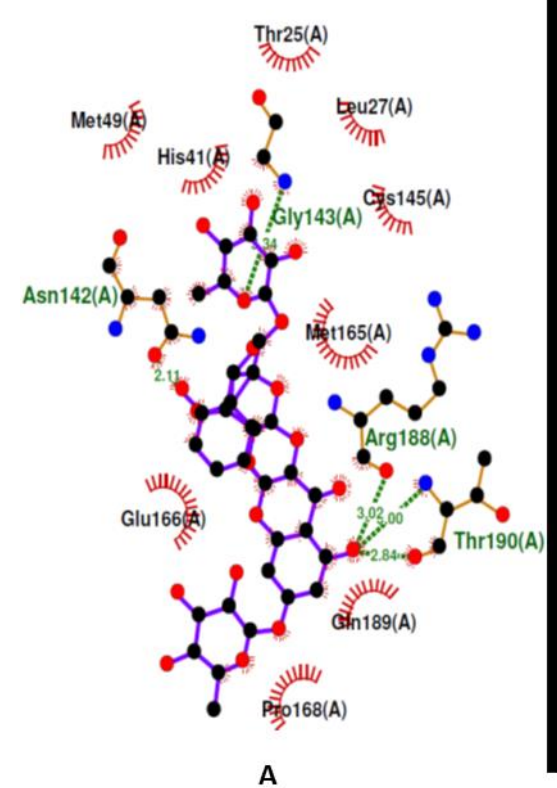

A

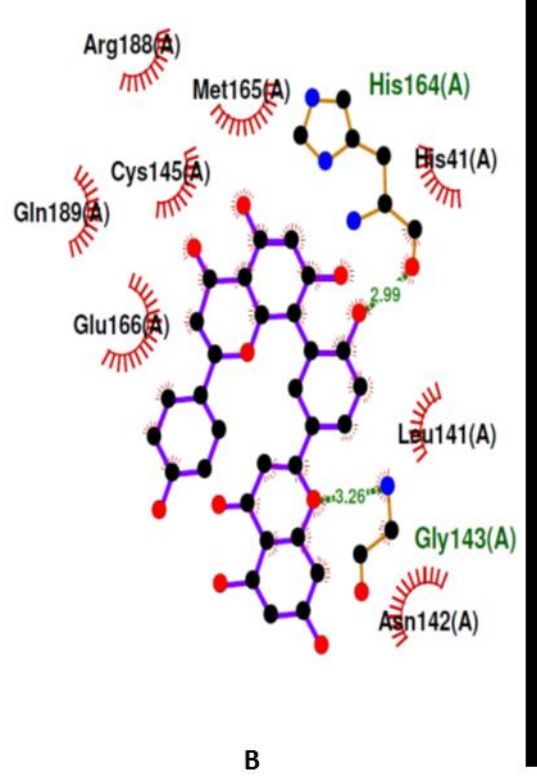

B

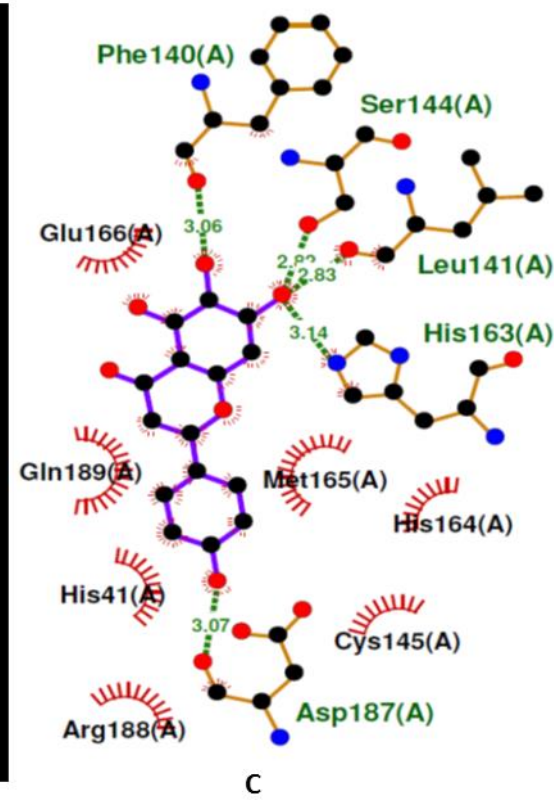

C

Figure 3. Interaction (2D) of particular amino acids in Mpro with ligand, robinin (A); amentoflavone (B); scutellarein (C). The interaction was plotted by LigPlot software. The respective amino acid formed hydrogen bonding colored in green, while the amino acids that are participated in the hydrophobic stabilization were indicated by black.

Free hydroxyl group particularly in A and B ring of flavonoids as reported to improve the inhibitory activity of flavonols against H1V-1 protease [19]. The glycosylation of kaempferol at C3 also showed an improved effect on its antiviral activity [24] which is related to the finding of this study for improving the binding affinity with Mpro by glycosylation at C-3. Another in silico study also revealed the glycosylation of C-7 improves the binding affinity of flavone with COVID-19 Mpro [25]. To further evaluate the potency of tested compounds as inhibitors of covid-19, the commercially available anti-HIV ritonavir which was approved by FDA as the covid-19 drug was also docked to the molecule to the protein. The docking value for ritonavir was $-7.2 \mathrm{kcal} / \mathrm{mol}$ which is lower than some of docked flavonoids molecules. That finding shows the potency of some selected natural flavonoids possessing anti-HIV as inhibitors for COVID-19 Mpro. To confirm the efficacy of the docked molecules, in vitro and in vivo experiments need to be addressed in the future. 


\subsection{ADME-tox and Drug-likeness analysis}

In the early stage screening of drug candidates, in-silico evaluation of ADME properties is majorly conducted to predict the pharmacological properties of drug candidates before conducting more laborious and expensive experiments such as in vitro and in vivo. To complete the understanding of selected flavonoids as potential SARS-Cov-2 Mpro inhibitors, the physicochemical properties linked to ADME (Absorption, Distribution, Metabolism, and Exertion), toxicity, and drug-likeness score were predicted using open source web tools as described in the methodology section. One of the widely accepted concepts for evaluating the ADME properties is the lipinski rule of 5 (Ro5). The Lipinski rule of five was originally comprehended to assist the development of orally bioavailable drug [26].

The predicted absorption properties were linked to orally available drugs which can be determined by several parameters such as coefficient partition (ClogP) properties, Polar Surface Area (PSA). Sugar substitution to flavonoid is lower lipophilicity properties as represented by ClogP of compounds 2, 5, 6, 14, and 15. The addition of sugar moiety increases the aqueous solubility. It increases the drug-likeness score of those compounds which was monitored higher than others without sugar. On the contrary, the addition of sugar moiety seemed to decrease the oral bioavailability of the compounds [27]. The ClogP is an important aspect to determine the absorption and permeability of drug candidates. The permissible value for the $\log \mathrm{p}$ is between -2 to 5 . The absorption percentage increase as the increment of $\log P$ within the permissible range. Drug candidates with Clog P between 0-3 are considered to have moderate bioavailability [28]. The ClogP also represents the lipophilicity of the molecule. If the $\log \mathrm{P}$ is too high, the drug has too low solubility in water, while too low $\log P$ could cause the difficulty of the drug to pass lipid bilayer [28]. The low bioavailability of sugar-linked flavonoids might be related to the low lipophilicity properties of the molecules that lead to difficulty to pass the lipid bilayer. The presence of more free hydroxyl decrease lipophilicity. All of the tested flavonoids without sugar moiety show moderate oral bioavailability properties. It is monitored that ligands with a relatively high number of $\mathrm{H}$-bond donors and acceptors are likely difficult for oral delivery showed by poor intestinal absorption properties [29]. Amentoflavone (10) showed the coefficient partition ahead of the permissible range $(>5)$, which indicates high lipophilicity of the compound. The bulky structure of amentoflavone might decrease the aqueous solubility. The unfavorable properties of amentoflavone (10) as drug candidate is also shown by both low drug-likeness and bioavailability scores.

Drug-likeness model score calculated by molsoft'st, was a combination effect of physicochemical, pharmacokinetics, pharmacodynamics properties of tested molecules [29]. İt also compared the tested molecules with the existing commercially available drug. A drug-likeness value is considered to be good if the value within the range 0.6-1.2 [8]. Almost all flavonoids have a positive drug-likeness value except for myricetin (9) and (-)-epigallocatechin (13). Both compounds possess a hydroxyl group in the B ring at C-3' which is not present in other tested ligands. The effect of $\mathrm{C}^{\prime}{ }^{\prime}-\mathrm{OH}$ to decrease the drug-likeness model need to be investigated further. The violation of Ro5 was exhibited by flavonoids with sugar moiety. Contradictory with the drug-likeness model score for flavonoids linked to sugar moiety show a higher value than the other without sugar. Although robinin (6) shows violation for the Ro5, the drug-likeness of robinin (6) showed the maximum among other ligands (drug-likeness score:1 ). This finding proposed that robinin (6) is likely unfavorable to administered orally according to its poor absorption properties but still has good potency as a therapeutic agent as shown by good drug-likeness.

This study revealed the relationship between the molecular weight of the ligand and the ability to permeate BBB (Blood Brain Barrier). Small molecule ligands demonstrated a better BBB score compared to the bigger ligand. The analysis described that drugs with $\mathrm{MW}<450 \mathrm{~g} / \mathrm{mol}$ are suggested to have better BBB permeation and tend to have good oral absorption characteristics [28]. The low BBB and oral bioavailability of flavonoids linked to the sugar moiety could be also connected to PSA (polar surface area) value. Polar surface area is important to predict drug transport properties [29]. The addition of sugar moiety obviously increases the PSA value above the acceptable range. The molecule with PSA higher than $140 \mathrm{~A}$ tends poorly permeate to membrane cells [30]. The BBB value range was determined 0-6, with 6 indicating the high BBB properties [31].

Though the pharmacokinetics profile is a significant part of drug development, it is also pivotal to consider the toxicity. lipophilicity is also related to toxicity. Since toxicity is a major factor leading to drug attrition at the discovery and development phases, it is important to evaluate this characteristic. The predicted acute toxicity in rats was calculated as lethal dose value run by Pro-Tox II webtool. According to table 2, almost 
all ligands exhibited relatively low acute toxicity represented by a high lethal dose value. The majority of the ligands are classified with unconcerned acute toxicity levels (levels 5 and 6). The exception is applied for quercetin (1) and myricetin (9) which are considered to have concerned acute toxicity with lethal dose appeared at $159 \mathrm{mg} / \mathrm{kg}$. Moreover, the in vivo study reported by Chen et.al also revealed the potential toxicity of quercetin (1) in the mice exposed by $100 \mathrm{mg} / \mathrm{kg}[32,33]$. Since toxicity is one of the important parameters in the drug design, several approaches such as side-group modifications are suggested to reduce the toxicity effect of the potential drug candidate.

Table 2. Table ADME properties, predicted bioavailability, drug-likeness score, and toxicity of ligands.

\begin{tabular}{|c|c|c|c|c|c|c|c|c|c|c|}
\hline \multirow[b]{2}{*}{ Ligands } & \multicolumn{5}{|c|}{ ADME properties } & \multirow{2}{*}{$\begin{array}{l}\text { Viola- } \\
\text { tion* }\end{array}$} & \multirow{2}{*}{$\begin{array}{l}\text { Bio- } \\
\text { availability }\end{array}$} & \multirow{2}{*}{$\begin{array}{l}\text { Drug- } \\
\text { likeness }\end{array}$} & \multirow{2}{*}{$\begin{array}{l}\text { LD } \\
(\mathrm{mg} / \mathrm{kg})^{* *}\end{array}$} & \multirow[b]{2}{*}{$\mathbf{B B B}^{* * *}$} \\
\hline & $\begin{array}{l}\text { H- } \\
\text { don(a) }\end{array}$ & $\begin{array}{l}\mathrm{H}- \\
\operatorname{acc}^{(b)}\end{array}$ & $C \log P$ & $\begin{array}{l}\text { MW } \\
(\mathrm{g} / \mathrm{mol})\end{array}$ & $\begin{array}{l}\text { PSA } \\
(\AA)\end{array}$ & & & & & \\
\hline 1 & 5 & 7 & 1.99 & 302.24 & 131.36 & 0 & 0.55 & 0.5 & 159 (c) & 2.55 \\
\hline 2 & 8 & 12 & -0.54 & 464.38 & 210.51 & 2 & 0.17 & 0.68 & $5000(\mathbf{e})$ & 1.66 \\
\hline 3 & 5 & 7 & 1.99 & 302.24 & 131.36 & 0 & 0.55 & 0.46 & 3919 (e) & 2.54 \\
\hline 4 & 4 & 6 & 2.28 & 286.24 & 111.13 & 0 & 0.55 & 0.5 & 3919 (e) & 2.78 \\
\hline 5 & 10 & 16 & -1.69 & 610.52 & 269.43 & 3 & 0.17 & 0.91 & 5000 (e) & 1.21 \\
\hline 6 & 11 & 19 & -2.89 & 740.66 & 308.12 & 3 & 0.17 & 1 & $5000(\mathrm{e})$ & 1 \\
\hline 7 & 4 & 6 & 2.28 & 286.24 & 111.13 & 0 & 0.55 & 0.38 & 3919 (e) & 2.75 \\
\hline 8 & 4 & 5 & 2.28 & 272.25 & 97.99 & 0 & 0.55 & 0.43 & 1000 (d) & 2.88 \\
\hline 9 & 6 & 8 & 1.69 & 318.24 & 151.59 & 1 & 0.55 & -0.24 & 159 (c) & 2.32 \\
\hline 10 & 6 & 10 & 5.13 & 538.46 & 181.8 & 2 & 0.17 & 0.19 & 3919 (e) & 0.55 \\
\hline 11 & 3 & 5 & 2.58 & 270.24 & 90.9 & 0 & 0.55 & 0.39 & 2500 (e) & 2.97 \\
\hline 12 & 4 & 6 & 2.28 & 286.24 & 111.13 & 0 & 0.55 & 0.43 & 3919 (e) & 2.78 \\
\hline 13 & 6 & 7 & 1.25 & 306.27 & 130.61 & 1 & 0.55 & -0.04 & 10000 (f) & 2.5 \\
\hline 14 & 7 & 14 & -0.8 & 592.5 & 217.19 & 3 & 0.17 & 0.7 & 5000 (e) & 1.24 \\
\hline 15 & 6 & 14 & -1.1 & 578.52 & 228.97 & 3 & 0.17 & 0.81 & 5000 (e) & 1.27 \\
\hline 16 & 5 & 6 & 1.55 & 290.27 & 110.38 & 0 & 0.55 & 0.64 & 10000 (f) & 2.73 \\
\hline
\end{tabular}

\subsection{Molecular Dynamic Simulation}

Molecular dynamic (MD) simulation is commonly used to evaluate atoms' behaviour, structural stability and to study the conformational changes on the atomic level. The structural flexibility of complex Mpro-robinin was exposed to MD to understand the stability of the complex in the water environment for the period of simulation. The complex of protein with robinin was chosen to be subjected to MD study since robinin has the highest binding affinity. Figure 4 displayed the time progression of RMSD value for protein and complex Mpro-robinin. The introduction of robinin to the binding cavity of Mpro did not give significant conformational changes to protein since two RMSD graphs are observed to be almost overlapping. Both systems reached equilibrium after 250 ps and showed relatively constant RMSD values for the remaining period. The range RMSD values of both systems are around 1.1 -2.3 $\AA$. A slight increment in RMSD value was observed between $500-650$ ps, showing RMSD value marginally over $2 \AA$. The fluctuation is happened due to the interaction of each residue, including electrostatic interaction, hydrogen bond, hydrophobic interactions, and water molecules which all contribute to structural re-arrangement at the interface of protein. The relatively stable RMDS value during MD simulation suggested that robinin is securely placed in the protein's binding pocket during simulation. This MD simulation strengthens the docking result describing the stability of the Mpro-robinin complex. 


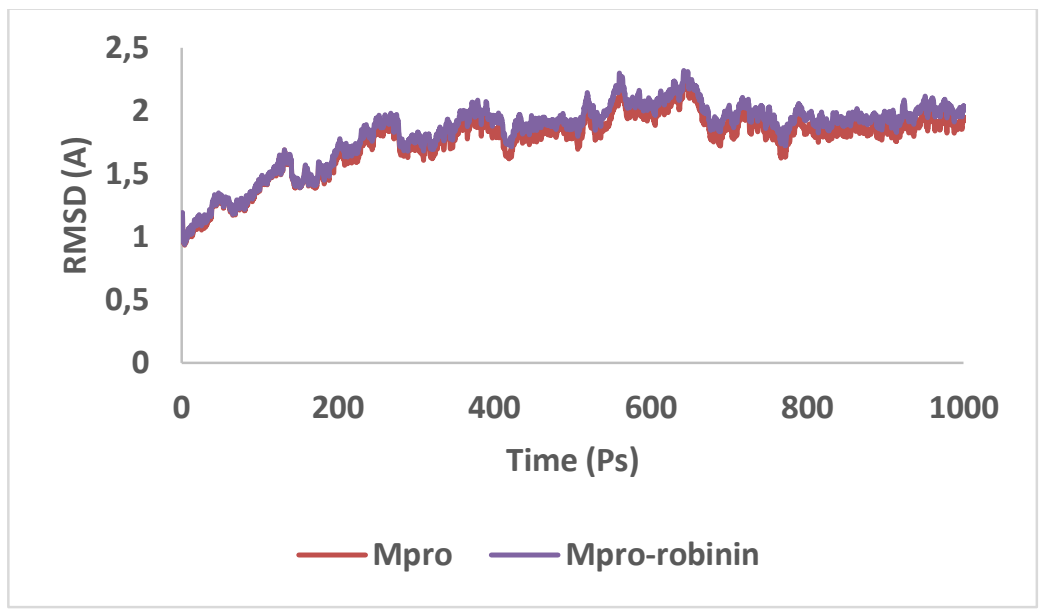

Figure 4. RMSD value of Mpro SASR-CoV-2 (red) and complex protein-robinin (purple) during MD simulation. RMSD obtained from MD analysis was conducted by VMD (Visual Molecular Dynamic) software.

RMSF measurement is used to analyze the local change in protein residue. The highest peak on RMSF graph (Figure 5) shows the most fluctuated protein region during simulation. RMSF value for both systems is within the range $0.42 \mathrm{~A}-2 \mathrm{~A}$ except for residue numbers 1 and 306. The narrow RMSF value indicates that the studied compounds kept close contact with their binding pockets during the MD simulations. Residues Ser1 (N-terminal) and Gln306 (C-terminal) show considerably high fluctuation, which is probably because both are located in the end-chain of protein and allow them to have more flexibility compared to other residues. Some residue with high RMSF values such as residue Ser46, Gly278, and Ala191 are known to construct the loop region. Both systems have RMSF values almost similar as shown by resembles phase of RMSF graph of protein and complex. The absence of different fluctuation in a specific area suggested the reasonable stability of protein after docking with robinin.

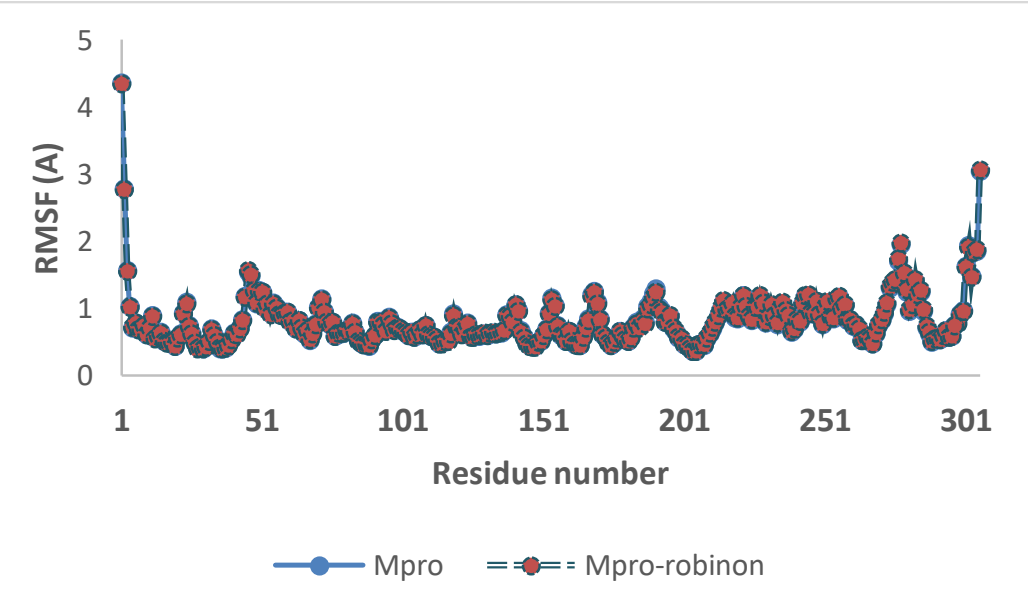

Figure 5. The RMSF value of Ca of Mpro (orange-red )and Mpro-robinin (blue) during simulation.

\section{CONCLUSION}

This study presented all of the tested molecules were suitably placed in of Mpro specific binding site with conserved catalytic dyad Cys145 and His41. Substitution of -OH with -OMe reduces the binding affinity in the C-4' in the flavone. The type of substituent in the C-7 and C-6 at A ring of flavone and flavonol group play an important role in the binding affinity. The position of free hydroxyl was also suggested to be important to improve the binding affinity. Among the tested molecules, robinin, a kaempferol-3-O-gal-rhm-7-O-rhm, showed the highest binding affinity $(-9 \mathrm{kcal} / \mathrm{mol})$ with hydrogen bindings are formed with Asn142; Gly143; Arg188; Thr190 amino acids. Robinin was also monitored to have higher than binding affinity to clinically approval Covid-19 drug, ritonavir $(-7.7 \mathrm{kcal} / \mathrm{mol})$, and lopinavir $(-8.2 \mathrm{kcal} / \mathrm{mol})$. The molecular dynamic 
study revealed the stability of the protein complex during the simulation. Moreover, the attachment of robinin to protein does not give significant conformational changes to the protein.

ADME properties and drug-likeness of molecules revealed flavonoids with sugar moiety displayed poor absorption properties. Low absorption leads to low oral bioavailability suggested the unfavorable properties for the development of the orally available drug. On the contrary, the calculated drug-likeness of flavonoids with sugar moiety was monitored higher than flavonoids without sugar. Robinin (8) was shown the highest drug-likeness score (value:1) among others. The toxicity result indicated that the majority of the selected flavonoids possess an un-concerned toxicity level. This study proposed robinin (8) as the most potential candidate for inhibiting SASR-CoV-2 Mpro. Despite possessing low oral bioavailability, robinin (6) shows a considerably good value for the binding affinity, predicted drug-likeness, and acute toxicity among tested molecules.

It should be concerned that these results were generated merely by an in silico investigation. Further advanced experiments are needed to test the efficacy of the tested compounds.

\section{MATERIALS AND METHODS}

\subsection{Ligand preparation}

Ligand structures were retrieved as SDF (3D) format from PubChem as the CID number was written in Table 1. Ligands were then converted into a PDB file using UCSF Chimera Molecular Visualization ( chimera-1.15 version) before it is proceeded into PDBQT files using Autodock tools for docking experiment. The Gasteiger charge was computed and the number of torsions was set not more than 8 for each ligand. The 3D structure of ligand number 8 (robinin) was nonavailable in the PubChem and was drawn manually by ChemBio3D Ultra 12.0 (www.cambridgesoft.com).

\subsection{Protein Preparation}

SARS-CoV-2 main protease (Mpro) with PDB id 6LU7 was downloaded from Protein Data bank (https ://www.rcsb.org/pdb/home/home.do). The protein preparation was completed by Autodock tools -1.5.6 application (http://autodock.scripps.edu/resources/tools) by removing the non-standard ligands in the protein structures. Hydrogen atoms were added to the structure before being saved in the PDB format for further treatment. The Gasteiger charges were automatically applied to the protein and then saved as chosen macromolecule in the PDBQT format.

\subsection{Docking Experiment}

The previously prepared ligands and protein were subjected to a docking experiment by first, formation the grid box for the docking process by AutodockTools. The ligand was placed in the center, the grid box was generated using $1 \AA$ spacing, the box size was $\mathrm{x}, \mathrm{y}, \mathrm{z} 14,20,12$, and the center was set at $\mathrm{x}$, $\mathrm{y}$, $9.768,11.436,68.904$ respectively. The grid box was constructed by the guidance of the coordinate of the cocrystalized ligand (N3 ligand) in the protein. The docking experiment was done by Autodock Vina 1.0 [34] with exhaustiveness value was set 8 . The rest of the parameters of the program were maintained as default in consideration for rigid protein and movable ligand. The docking result was identified as binding affinity energy. The Pymol 1.8.6.0 was used to visualize the docking result and also to calculate the RMSD. The used parameters were validated by redocking the native ligand into the protein and the RMSD value of the native ligand and re-docked native ligand was calculated. The interaction of ligands and protein in the complex was analyzed by LIGPLOT v.4.5.3 (www.ebi.ac.uk) [35].

\subsection{ADME properties and Drug-likeness of the ligands}

The Drug-likeness properties were described as the ADME (Adsorption, Distribution, Metabolism, and Exertion) properties accommodated the Lipinski rule of five, polar surface, and also the bioavailability. Those properties were evaluated by the Swiss ADME web tools (http://www.swissadme.ch/index.php). The toxicity profiles represented by lethal dose (LD50) for each ligand were predicted using the open-source web tool ProTox (http:// tox.charite.de/protox_II/). The possible drug-ability score for each ligand was calculated using the tool, Molsoft (http://molsoft.com/mprop/).

\subsection{Molecular Dynamic Simulation}

To confirm the stability of protein-ligand complex, the complex of robinin-Mpro was subjected to the MD by nanoscale molecular dynamics (NAMD) software [36]. The configuration files (parameters and topology) 
for MD CHARMM36 all-force field parameters are generated by a web-based CHARMM-GUI (http://www.charmm-gui.org/) [37] along with the VMD Force Field Toolkit (ffTK) [38]. All systems were solvated with transferable intermolecular potential water molecules (TIP3P) in cubic periodic boundary conditions with the size around $52 \times 67 \times 60$ Angstrom $(x, y, z)$. The counter ion (Na+ and $\mathrm{Cl}-)$ were added to neutralize the system. Before running the simulation, the energy of all systems was minimized in 500 steps to prevent steric clashes and identify stable conformation. The constant temperature control was based on Langevin dynamics 23 with a damping coefficient (gamma) of $1.0 \mathrm{ps}$. The full-system periodic electrostatics were computed by the particle-mesh Ewald (PME) algorithm24. The MD simulation was conducted for 1000 ps under the normal temperature $(310 \mathrm{~K})$ and pressure (1bar). The system is equilibrated with the NPT ensemble and the trajectory was recorded each 0.2 ps. Visual Molecular Dynamic (VMD) version 1.9.3 [38] was used to visualize and analyze the MD result. The root-mean-square deviation (RMSD) value was extracted from MD simulation to evaluate the stability of subjected protein-ligand complex.

Acknowledgments: This research was supported by the Research Center of Chemistry, National Research and Innovation Agency (BRIN) of Indonesia.

Author contributions: Concept, Data collection and processing and main contributor - L.O.; Supervision - A.A; Resources - A.A; Analysis and/or Interpretation - L.O. and A.A. Literature Search -L.O. and P.P., Writing - L.O.; Critical Reviews - A.A., L.O., P.P. The manuscript was edited and approved by all authors. All authors have responsibilities to the contents and writing of the manuscript.

Conflict of interest statement: The authors declared no conflict of interest in the manuscript.

\section{REFERENCES}

[1] Grant WB, Lahore H, McDonnell SL, Baggerly CA, French CB, Alliano J, Bhattoa HP. Evidence that vitamin d supplementation could reduce risk of influenza and covid-19 infections and deaths. Nutrients. 2020; $12(4): 988$. [CrossRef]

[2] Mouffouk C, Mouffouk S, Mouffouk C, Hambaba, Haba H. Flavonols as potential antiviral drugs targeting SARSCoV-2 proteases (3CLpro and PLpro), spike protein, RNA-dependent RNA polymerase (RdRp) and angiotensinconverting enzyme II receptor (ACE2). Elsevier B.V. 2021; 891: 173759. [CrossRef]

[3] Doremallen NV, Bushmaker T. Aerosol and Surface Stability of SARS-CoV-2 as Compared with SARS-CoV-1. N. Engl J Med. 2020; 382: 1564-1567. [CrossRef]

[4] Mousavizadeh L, Ghasemi S, Genotype and phenotype of COVID-19: Their roles in pathogenesis. J. Microbiol. Immunol. Infect. 2021; 54(2): 1-4. [CrossRef]

[5] Chang KO, Kim Y, Lovell S, Rathnayake AD, and Groutas WC. Antiviral drug discovery: Norovirus proteases and development of inhibitors. Viruses. 2019; 11(2): 1-14. [CrossRef]

[6] Gyebi GA, Ogunro OB, Adegunloye AP, Ogunyemi OM, Afolabi SO. Potential inhibitors of coronavirus 3chymotrypsin-like protease (3CLpro): an in silico screening of alkaloids and terpenoids from African medicinal plants. J Biomol Struct Dyn. 2020; 39(9): 3396-3408. [CrossRef]

[7] Goyal B and Goyal D, Targeting the Dimerization of the Main Protease of Coronaviruses: A Potential BroadSpectrum Therapeutic Strategy. ACS Comb Sci. 2020; 22(6): 297-305. [CrossRef]

[8] Swain SS, Singh SR, Sahoo A, Hussain T, Pati S. Anti-HIV-drug and phyto-flavonoid combination against SARSCoV-2: a molecular docking-simulation base assessment. J Biomol Struct Dyn. 2021; 1-14. [CrossRef]

[9] Rabby MII. Current drugs with potential for treatment of covid-19: A literature review. J Pharm Pharm Sci. 2020; 23(1): 58-64. [CrossRef]

[10] Khaerunnisa S, Kurniawan H, Awaluddin R, Suhartati S. Potential Inhibitor of COVID-19 Main Protease ( M pro ) from Several Medicinal Plant Compounds by Molecular Docking Study. Preprints. 2020: 1-14. [CrossRef]

[11] Lokhande KB, Doiphode S, Vyas R, Swamy KV. Molecular docking and simulation studies on SARS-CoV-2 Mpro reveals Mitoxantrone, Leucovorin, Birinapant, and Dynasore as potent drugs against COVID-19. J Biomol Struct Dyn. 2020; 1-12. [CrossRef]

[12] Mahdi M, Mótyán JA, Szojka ZI, Golda M, Miczi M, Tózsér M. Analysis of the efficacy of HIV protease inhibitors against SARS-CoV-2's main protease. Virol J. 2020; 17(1): 190. [CrossRef] 
[13] Zhou et al. A pneumonia outbreak associated with a new coronavirus of probable bat origin. Nature. 2020; 579(7798): 270-273. [CrossRef]

[14] Kraus M, Bader J, Overkleeft H, and Driessen C. Nelfinavir augments proteasome inhibition by bortezomib in myeloma cells and overcomes bortezomib and carfilzomib resistance. Blood Cancer J. 2013; 3(3): 1-9. [CrossRef]

[15] Zhong DS et al. HIV protease inhibitor ritonavir induces cytotoxicity of human endothelial cells. Arterioscler Thromb Vasc Biol. 2002; 22(10): 1560-1566. [CrossRef]

[16] Saravanan D, Thirumalai D, and Asharani IV. Anti-HIV flavonoids from natural products: A systematic review. Int J Res Pharm Sci. 2015; 6(3): 248-255.

[17] Ko YJ, Oh HJ, Ahn HM, Kang HJ, Kim JH, Ko YH.Flavonoids as Potential Inhibitors of Retroviral Enzymes. J Korean Soc Appl Biol Chem. 2009; 52(4): 321-326. [CrossRef]

[18] Lee JS , Kim HJ, Lee YS. A new Anti-HIV Flavonoid Glucuronide from Chrysanthemum morifolium. Planta Med. 2003; 69(9): 859-861. [CrossRef]

[19] Xu HX, Wan M, Dong H, Hay-But PP, Foo LY. Inhibitory activity of flavonoids and tannins against HIV-1 protease. Biol Pharm Bull. 2000. 2000; 23(9):1072-1076. [CrossRef]

[20] Owis AI, El-Hawary MS, Amir ED, Aly OM, Abdelmohsen UR, Kamel MS. Molecular docking reveals the potential of Salvadora persica flavonoids to inhibit COVID-19 virus main protease. RSC Adv. 2020; 10(33): 19570-19575. [CrossRef]

[21] Zakaryan H, Arabyan E, Oo A, Zandi K. Flavonoids: promising natural compounds against viral infections. Arch Virol. 2017; 162(9): 2539-2551. [CrossRef]

[22] Phosrithong N, Ungwitayatorn J. Molecular docking study on anticancer activity of plant-derived natural products. Med Chem Res. 2010; 19(8): 817-835. [CrossRef]

[23] Áy É, Hunyadi A, Mezei M, Minárovits J, Hohmann J. Flavonol 7- O -Glucoside Herbacitrin Inhibits HIV-1 Replication through Simultaneous Integrase and Reverse Transcriptase Inhibition. Evidence-based Complement. Altern Med. 2019; 2019: 1-6. [CrossRef]

[24] Pastor N, Collado MC, Manzoni P. Phytonutrient and nutraceutical action against COVID-19: Current review of characteristics and benefits. Nutrients. 2021; 13(2): 1-10. [CrossRef]

[25] Omrani M, Bayati M, Mehrbod P, Bardazard KA, Nejad-ebrahimi S. Natural products as inhibitors of COVID-19 main protease - A virtual screening by molecular docking. Pharm Sci. 2021; 27(Suppl 1): 1-37. [CrossRef]

[26] Lipinski CA, Lombardo F, Dominy BW, and Feeney FJ. Experimental and computational approaches to estimate solubility and permeability in drug discovery and development settings. Adv Drug Deliv Rev. 2012; 64(2012): 417. [CrossRef]

[27] Oktavia L, Krishna VS, Rekha EM, Fathoni A, Sriram D, Agusta A. Anti-mycobacterial activity of two natural Bisanthraquinones: (+)-1,1'-Bislunatin and (+)-2,2'-Epicytoskyrin A. IOP Conf Ser Earth Environ Sci. 2000; 591(2020): 1-14. [CrossRef]

[28] Zerroug A, Belaidi S, BenBrahim I, Sinha L, Chtita S. Virtual screening in drug-likeness and structure/activity relationship of pyridazine derivatives as Anti-Alzheimer drugs. J King Saud Univ - Sci. 2019; 31(4): 595-601. [CrossRef]

[29] Chandrasekaran K, Kumar RT. Molecular properties prediction, docking studies and antimicrobial screening of ornidazole and its derivatives. J Chem Pharm Res. 2016; 8(3):849-861.

[30] Schaftenaar G, Vlieg JD. Quantum mechanical polar surface area. J Comput Aided Mol Des. 2012; 26: 311-318. [CrossRef]

[31] Gupta M, Lee HJ, Barden CJ , Weaver DF. The Blood-Brain Barrier (BBB) Score. J Med Chem. 2019; 62(21): 98249836. [CrossRef]

[32] Chen $\mathrm{R}$ et al. Potential toxicity of quercetin: The repression of mitochondrial copy number via decreased POLG expression and excessive TFAM expression in irradiated murine bone marrow. Toxicol Reports. 2014; 1: 450-458. [CrossRef]

[33] Choi KY, Liu G, Lee S, Chen X. Theranostic nanoplatforms for simultaneous cancer imaging and therapy: current approaches and future perspectives. Nanoscale. 2012; 4(2): 330-342. [CrossRef]

[34] Trott O, Olson AJ. AutoDock Vina: Improving the speed and accuracy of docking with a new scoring function, efficient optimization, and multithreading. J Comput Chem. 2009; 31(2): 455-461. [CrossRef] 
[35] Wallace AC, Laskowski RA, Thornton JM. LIGPLOT: a program to generate schematic diagrams of protein-ligand interactions. Protein Eng Des Sel. 1995; 8(2): 127-134. [CrossRef]

[36] Phillips JC et al. Scalable molecular dynamics with NAMD. J ComputChem. 2005; 26(16): 1781-1802. [CrossRef]

[37] Jo S, Kim T, Iyer VG, Im W. CHARMM-GUI: a web-based graphical user interface for CHARMM.J Comput Chem. 2008; 29(11): 1859-1865. [CrossRef]

[38] Humphrey W, Dalke A, Schulten K. VMD: Visual molecular dynamics. J Mol Graph. 1996; 14(1): 33-38. [CrossRef] This is an open-access article that is publicly available on our journal's website under Institutional Repository at http://dspace.marmara.edu.tr. 